\title{
Association of Terminal Complement Complex (TCC), Prostaglandin (PGj2) among Patient with First Episode Psychosis (Case-Control Study)
}

\author{
Rana Fadhil Obaid ${ }^{1}$, Rasha Fadhel Obaid ${ }^{2}$, Sumeiya Mahdi al Har ${ }^{3}$ \\ ${ }^{1}$ Lecturer in Department of Medical Microbiology, College of Medicine, University of Kufa, Najaf, Iraq, ${ }^{2}$ Lecturer \\ in Department of Anesthesia Techniques, Al-Mustaqbal University College, Babylon, Iraq, ${ }^{3}$ Assistant lecturer in \\ Department of Medical Microbiology, Jabir ibn Hayyan Medical University, Najaf, Iraq,
}

\begin{abstract}
The purpose of the study investigate the association between some inflammatory biomarkers with first episode psychosis (FEP) and to find out the correlation with different socio demographic profile. A casecontrol study was involve patients with first episode psychosis and healthy control. peripheral Blood samples collected from 44 cases they were attending AL-Hakeem General hospital, Psychiatric Department, Najaf, Iraq, in the period between January, 2019 to may, 2019. The cases included females and males, and the age was 18-70 years. Control: 44 healthy controls who had no history or clinical evidence of first episode psychosis or any other disease. The result revealed that increase in the level of serum Complement Complex C5b-9 and decrease Prostaglandin J-2 among FEP patients (1021.7 \pm 58.5 and 3.82 \pm 0.36 ) respectively in comparison to control were mean $(585.87 \pm 47.059$ and $9.94 \pm 0.67)$. The results of this research concluded that, the inflammatory response is an immune system that allow the individual to cope with various menacing advise, but in long-lasting conditions and pathological, the continuous of this stimulate could develop into harmful. The regulation of the all development involves endogenous counter-balancing mechanisms that control special effects of deleterious pro inflammatory mediators. information showing a misbalance in some pro inflammatory/anti inflammatory in blood of person with FEP.
\end{abstract}

Keywords: first episode psychosis, serum Complement Complex C5b-9, Prostaglandin J 2. Psychosis.

\section{Introduction}

The word first episode psychosis (FEP) is applied to describe conditions that impact the mind, in which people have problems distinguishing between what is true and what is not. When this occurs, it is called a psychotic episode. The pathogenesis and etiology of FEP is understood, although the facts suggests a contribution from both genetic factors and environmental. ${ }^{[1]}$

The complement system is a essential part of the innate immune defense, but it is also implicated in the induction of the adaptive immune response and in the removal of dead cells and immune complexes. Also, it helps the ability of phagocytic cells and antibodies to clear pathogens or antigens from an organism. Alters in the level of complement system can be discovered in FEP in compare with healthy. The measurement in serum concentration from both of a selected panel of complement and activation compound with the aim of gaining insight into identifying candidate biomarkers and underlying pathology. ${ }^{[2]}$

The Prostaglandin 15d-PGJ2 is the newly discovered type of prostaglandin. dehydration product of PGD2, unlike other prostaglandins in numerous respects. 15d-PGJ2 production by no specific prostaglandin synthase (PGS) and no specials 15d-PGJ2 receptor has been well-known to date. In place of, 15d-PGJ2 worked by PGD2 receptors. These characters are accountable for numerous of anti inflammatory functions. ${ }^{[3]}$

In the deficiency of a biological marker, the diagnosis relies upon clinical assessment. Besides participate to difficulties in early diagnosis, therapeutic choice, disease stratification, (treatment is largely experimental 
and prediction of outcome). ${ }^{[4]}$

The purpose of the study was to examine the role for using complement-related proteins (Terminal complement complex C5B-9) and Prostaglandin J2(PGJ2) as serum biomarkers for FEP. However, the biomarkers could help to explain the pathogenesis of the disease and the present of biomarkers for FEP which would be supportive for diagnosis and might help to know the molecular basis for these conditions, in the outlook studies, to be directly concerned in causing the disease symptoms, they would be very important targets for prevention efforts and rational treatment. ${ }^{[5]}$

Methods: Study Design : Case-control study:

Patients: The study population involve patients with first episode psychosis. peripheral Blood samples collected from 44 cases at AL-Hakeem General hospital, Psychiatric Department, in Najaf, Iraq in the period between January, 2019 to May 2019, and the age was 18-70 years.

Control: 44 healthy controls who had no history or clinical evidence of first episode psychosis or any other disease.

Inclusion criteria: First episode psychotic patients drug naïve.

Exclusion criteria: All patients with any acute or chronic diseases and patients with autoimmune disease were excluded from this study in addition excluding pregnant women.

Informed consent: Informed consent was obtained from all individual participants included in the study.

Samples of blood were taken from the individuals enrolled in this study after obtaining the oral consent of them. $5 \mathrm{ml}$ of blood was obtained from cases and controls but, before drawing the blood, had to be cleaned the skin and blood sample were distributed to $2 \mathrm{ml}$ of blood to EDTA tube that for (ESR and WBC) and $3 \mathrm{ml}$ of blood to plain tube for immunological tests the centrifuge samples at $2-8^{\circ} \mathrm{C} 2000-3000$ Round Per/Minutes for 15 minutes within 30 minutes.

Study Parameters: All suspected patients first episode psychosis and control group subjected to the following immunological tests; Complement complex TCC by ELISA, Prostaglandin J2 by ELISA and Serological Tests Erythrocyte Sedimentation Rate (ESR), White Blood Cell Count.

Statistical Analysis: The data of present study were articulated as Mean \pm Standard Error, the statistical analysis Descriptive statistics, Correlation coefficients, P-value were using Graphpad prism to calculated. The comparison between two groups were analyzed by t-test and the comparison among subdivided. when P-value $<$ 0.05 was statistically a significant relations between 2 categorical variables was explored by cross- tabulation. The statistical significance of such associations was assessed by Chi-square ( $\chi 2)$ test. An estimate was considered statistically significant if its $\mathrm{P}$ value was less than an $\alpha$ level of significance of 0.05 .

\section{Findings}

Forty four patients with FEP and forty four healthy control enrolled in the present study, Table (1) shows the mean of ESR to patient (13.7) control (9.6) and WBC count to patient (10209), control (7847).

\section{Collection of Sample}

Table (1): Mean of Erythrocyte sedimentation rate and white blood cell count in the studied group

\begin{tabular}{|c|c|c|c|}
\hline \multirow{2}{*}{ Hematological tests } & Cases & Controls $N=44$ & \multirow{2}{*}{ Normal range } \\
\hline & \multicolumn{2}{|c|}{ Mean \pm SE } & \\
\hline ESR & 13.7 & 9.6 & $(5-20)$ \\
\hline WBC & 10209 & 7847 & $(4000-7000)$ \\
\hline
\end{tabular}


The result of table (2) revealed that increase in the level of serum Complement Complex C5b-9 and decrease PGJ2 among FEP patients $(1021.7 \pm 58.5$ and $3.82 \pm 0.36)$ respectively in comparison to control were mean $(585.87 \pm 47.059$ and 9.94 \pm 0.67$)$ respectively. Moreover there was a significant differences $(\mathrm{P}<0.05)$ among cases \& control in regard to concentration of C5b-9, and in serum PGJ-2 levels in patients with FEP in comparison with healthy group.

Table(2) Distribution of C5b-9 and PGJ2 in the Studied Groups.

\begin{tabular}{|l|l|l|l|}
\hline \multirow{2}{*}{ Biomarkers } & \multicolumn{2}{|l|}{ FEP N=44 } & \multicolumn{2}{l|}{ Controls N=44 } & P value \\
\cline { 2 - 4 } & \multicolumn{1}{|l|}{ Mean \pm SE } & $585.87 \pm 47.059$ & \\
\hline C5b9 & $1021.7 \pm 58.5$ & & 0.00106 \\
\hline PGJ2 & $3.82 \pm 0.36$ & $9.94 \pm 0.67$ & 0.000119 \\
\hline
\end{tabular}

\section{Comparison serum biomarkers levels according to gender in patients .}

Table (3) indicated there is no significant differences ( $p>0.05)$ in biomarkers concentration (C5b-9 and PGJ2) among patients with FEP according to gender where the concentration of biomarkers PGJ2 and C5b-9 among male were ( 3.97 and 1046.7) respectively in comparison to female were ( Assistant Lecturer, Faculty of Physical Therapy, Modern University for Technology and Information and 1008.3 ) respectively.

Table (3) Comparison serum biomarkers concentration according to gender in patients.

\begin{tabular}{|c|c|c|c|}
\hline \multirow{2}{*}{ Markers } & \multicolumn{2}{|l|}{ Mean \pm S.E. } & \multirow{2}{*}{ p-value } \\
\hline & Male & Female & \\
\hline PGJ2 & $3.97 \pm 0.36$ & $3.737 \pm 0.33$ & 0.35 \\
\hline C5B-9 & $1046.7 \pm 82.8$ & $1008.3 \pm 45.45$ & 0.25 \\
\hline
\end{tabular}

Comparison serum biomarkers concentration according to Age in patients.

The results of biomarkers levels (C5b-9 and PGJ2) in FEP patients reflect no significant differences ( $\mathrm{p}>0.05)$ according to age groups, as shown in table (4). The table also shows a marked decrease in the level or concentration of PGJ2 in comparison to level of the C5b-9.

Table (4) The mean of serum biomarkers concentration in FEP patients according to age.

\begin{tabular}{|l|l|l|l|}
\hline \multirow{2}{*}{ Markers } & \multicolumn{2}{|l|}{ Mean \pm S.E. } & \multirow{2}{*}{ p-value } \\
\cline { 2 - 4 } & $\mathbf{( 1 5 - 2 9 )} \mathbf{~ N = 1 8}$ & $\mathbf{( 3 0 - 5 5 )} \mathbf{N}=\mathbf{2 6}$ & \\
\hline PGJ2 & $3.1 \pm 0.42$ & $4.3 \pm 0.24$ & 0.31 \\
\hline C5b-9 & $1028.4 \pm 71.7$ & $1016.3 \pm 47.1$ & 0.12 \\
\hline
\end{tabular}


Estimate the concentration of the biomarkers (C5b-9 and PGJ2) among FEP patients in regards to occupation show statically insignificant ( $p>0.05)$, the mean value of C5b-9 and PGJ2 among employed were $(3.76$ and $1,101.57)$ in comparison among unemployed (4.15 and 929.3) respectively. Table (5).

Table (5) Comparison serum biomarkers levels according to occupation in patients.

\begin{tabular}{|c|c|c|c|}
\hline \multirow[t]{2}{*}{ Markers } & \multicolumn{2}{|l|}{ Mean \pm S.E. } & \multirow{2}{*}{ p-value } \\
\hline & Employed N=28 & Unemployed $\mathrm{N}=16$ & \\
\hline PGJ2 & $3.76 \pm 0.29$ & $4.15 \pm 0.51$ & 0.31 \\
\hline C5b-9 & $1,101.57 \pm 61.83$ & $929.3 \pm 19.9$ & 0.14 \\
\hline
\end{tabular}

\section{Comparison serum biomarkers concentration according to residence in patients}

Distribution of concentration of the biomarkers (C5b-9 and PGJ2) among FEP patients in regards to residence reflect no significant differences $(p>0.05)$ among the mean of the biomarkers in urban and rural as shown in table (6).

Table (6) Comparison serum biomarkers levels according to residence in patients.

\begin{tabular}{|c|c|c|c|}
\hline \multirow{2}{*}{ Markers } & \multicolumn{2}{|l|}{ Mean \pm S.E. } & \multirow[b]{2}{*}{ p-value } \\
\hline & Urban & Rural & \\
\hline PGJ2 & $3.95 \pm 030$ & $3.68 \pm 0.4$ & 0.22 \\
\hline C5b-9 & $994.8 \pm 54.9$ & $1,106.3 \pm 165.9$ & 0.55 \\
\hline
\end{tabular}

The correlation to determine the association between PGJ2 and C5b-9, with first episode psychosis diseases, the figure (1) reflect that, there is a inverse (negative) correlation $(r=-0.249)$ between C5b-9 and PGJ2 among FEP patients.

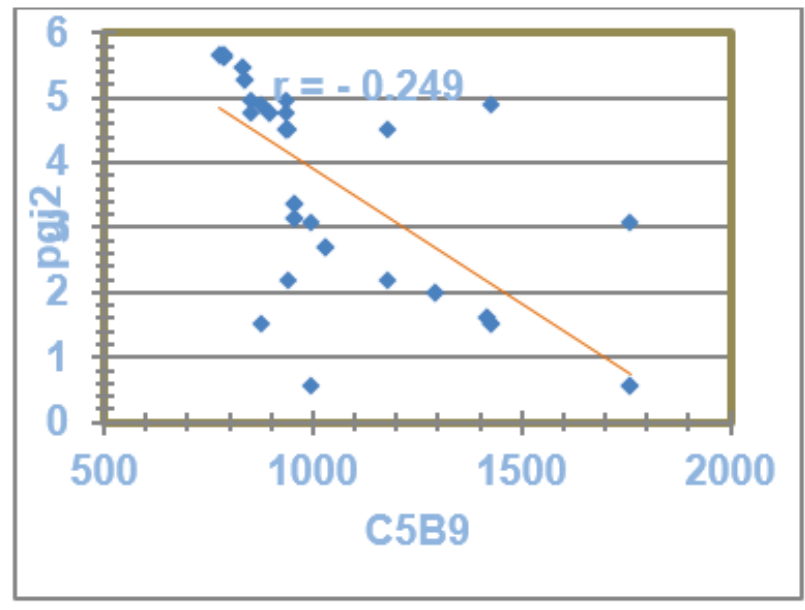

Fig 1 the graphical presentation of the inverse correlation between C5b-9and PGJ2 in patients with FEP r = - 0.249. 


\section{Discussion}

Distribution of Complement Complex C5b-9 and PGJ-2, in the Studied Groups.

In this study the concentration of the terminal complement complex (C5b-9) was investigated in serum of 44 patients with FEP and 44 healthy controls by using ELISA kit and the results showed that the p-value $=0.001$. The result of the following studies are similar to and support the result of our study ${ }^{[6,7,8]}$ they said the association between $(\mathrm{C} 5 \mathrm{~b}-9)$ and disease $\mathrm{p}$ value $=$ 0.003 .

In this current study the concentration of the PGJ-2 was investigated in serum of 44 patients with FEP and 44 healthy controls by using ELISA kit and the results showed that the decrease in concentration PGJ-2 in patients with FEP compared with the control p-value $=0.001$. The results of current study are in agreement with results reported by. ${ }^{[9,10]}$. There is decrease in the concentration of pgj2 biomarker in patients due to the presence of inflammation consumes the natural concentration of marker, but in control ratios were normal.

\section{Comparison serum biomarkers levels according to gender in patient and control}

The results in this study indicated there is no significant differences $(p>0.05)$ in comparison serum biomarkers concentration (C5b9 p-value $=0.35$, PGJ-2 $\mathrm{p}$-value $=0.25$ ) among patients with FEP according to gender. This result agreement with results reported by ${ }^{[11]}$ but find in some study the significant result. ${ }^{[12]}$

The probable explanations of these findings is that female and male FEP are depending on specific clinical observations, the environmental influences such as smoking, which are more often present in men.

\section{Comparison serum biomarkers levels according} to age in patients and controls.

The results in this study indicated there is no significant differences $(p>0.05)$ in comparison serum biomarkers concentration (C5b9 p-value $=0.12, \mathrm{PGJ}-2$ p-value $=0.31)$ in patients with FEP according to age. The result of this study agreement with the study by [13] they reported no significant differences $(p>0.05)$.
In another studies by. ${ }^{[14]}$ Significant differences $(p$ $<0.05)$. The effects and pressures of social and psychological impact on all ages, adding to the circumstances of war and death and orphanhood and unemployment and others.

\section{Comparison serum biomarkers concentration} according to occupation in patients.

The results of this study indicated there is significant differences $(p<0.05)$ in comparison serum biomarkers concentration in patients with FEP according to occupation group.

The result of this study supported by previous studies like, ${ }^{[15]}$ while other studies reported statistically no signifcant differences like. ${ }^{[16,17]}$. The result of the current study reported there is significant differences because the exposure to work problems and may be associated with other socio demographic characters.

\section{Comparison serum biomarkers concentration} according to residence in patient

The results of this study indicated there is no signifcant differences $(p>0.05)$ in comparison serum biomarkers concentration (C5b-9p-value $=0.55$,) in patients with FEP according to residence, in the previous studies showing similar result. ${ }^{[18,19]}$ while in another studies find signifcant result. ${ }^{[20,21]}$ There is no difference in results because rural life at present is very similar to the advanced life in the city and so they are subject to the same influences.

\section{Results of the correlation coefficient among biomarkers in patients with FEP}

The result of figure (1) inverse (negative) correlation between C5b-9 andPGJ2 among FEP patients. in the previous studies showing same result. $[22,23]$ The inflammatory response is an immune system that allow the individual to cope with various menacing advise, but in long-lasting conditions and pathological, the preservation of this stimulate could develop into harmful. The regulation of the all development involves endogenous counter-balancing mechanisms that control special effects of deleterious pro inflammatory mediators. Information showing a misbalance in some pro inflammatory/anti inflammatory in blood of person with FES. ${ }^{[24]}$ 


\section{Conclusion}

- The inflammation association with symptoms of First Episode Psychosis.

- All age groups are at risk of having an equal incidence of First Episode Psychosis.

- Males and females have the same chance of becoming First Episode Psychosis.

- Biomarkers PGJ2 and C5b9 can be used as diagnostic criteria.

Conflict of Interest: there is no conflict of interest.

Ethical approval: This study protocol was accepted by the ethical committee, College of Medicine University of Kufa.

\section{Source of Funding: Self}

\section{References}

1. Busse S, Busse M, Schiltz K, Bielau H, Gos T, Brisch $\mathrm{R}$ et al. Different distribution patterns of lymphocytes and microglia in the hippocampus of patients with residual versus paranoid schizophrenia: further evidence for disease courserelated immune alterations?. J Clin Psychiatry $2011 ; 63: 420-4$.

2. Charles A Janeway, Jr, Paul Travers, Mark Walport, and Mark J Shlomchik. The Immune System in Health and Disease. Text book of Immunobiology, 5th edn. New York: Garland Science; 2001 ISBN10: 0-8153-3642-X.

3. Scher $J U$ and Pillinger $M H$. The anti-inflammatory effects of prostaglandins. Am J Psychiatry 2009;16:1-56.

4. Weickert CS, Weickert TW, Pillai A, Buckley PF. Biomarkers in schizophrenia: a brief conceptual consideration. Dis Markers 2013;35: 3-9.

5. Wang AK and Miller B J. Meta-analysis of cerebrospinal fluid cytokine and tryptophan catabolite alterations in psychiatric patients: comparisons between schizophrenia, bipolar disorder, and depression. Schizophr Res 2017;44:75-83.

6. Mueser KT, Penn DL, Addington J, Mary FB, Susan G, Shirley MG et al. The navigate program for first-episode psychosis: rationale, overview, and description of psychosocial components. Psychiatr Serv. 2015;66:680-90.

7. Pouget JG, Goncalves VF, Schizophrenia WGotPGC, Spain SL, Finucane HK, Raychaudhuri $\mathrm{S}$ et al. Genome wide association studies suggest limited immune gene enrichment in schizophrenia compared to 5 autoimmune diseases. Schizophr Bull 2016; 42:1176-84.

8. Pettingill P, Kramer HB, Coebergh JA, Pettingill R, Maxwell S, Nibber A, et al. Antibodies to GABAA receptor $\alpha 1$ and $\gamma 2$ subunits: clinical and serologic characterization. Neurology 2015;84: 1233-41.

9. Schneider M, Debbane M, Bassett AS, Chow EW, Fung WL, van den Bree M et al. Psychiatric disorders from childhood to adulthood in 22q11.2 deletion syndrome: results from the International Consortium on Brain and Behavior in 22q11.2 Deletion Syndrome. Am J Psychiatry 2014; 171:627-39.

10. Bloomfield PS, Selvaraj S, Veronese M, Rizzo G, Bertoldo A, Owen DR, et al. Microglial activity in people at ultra high risk of psychosis and in schizophrenia: an [11C]PBR28 PET brain imaging study. Am J Psychiatry 2016;173: 44-52.

11. Stowell SR, Winkler AM, Maier CL, Arthur CM, Smith NH, Girard-Pierce KR, et al. Initiation and regulation of complement during hemolytic transfusion reactions. Clin Dev Immunol 2012; 307093.

12. Mäntylä $T$, Mantere $O$, Raij TT, Kieseppä $T$, Laitinen H, Leiviskä J, etal. Altered activation of innate immunity associates with white matter volume and diffusion in first-episode psychosis. PLoS One 2015;10:e0125112.

13. Fukushima M. Biological activities and mechanisms of action of PGJ2 and related compounds: an update. Prostaglandins Leukot Essent Fatty Acids 1992;47:1.

14. Liu Z, Tang Q, Wen J, Yan T, DaMin H, Yuzhen $\mathrm{H}$, etal. Elevated serum complement factors 3 and 4 are strong inflammatory markers of the metabolic syndrome development: a longitudinal cohort study. Sci Rep 2016;6:18713.

15. Prasad K, Upton C, Nimgaonkar V, Keshavan M. Differential susceptibility of white matter tracts to inflammatory mediators in schizophrenia: An integrated DTI study. Schizophr Res 2015; 161:119-25. 
16. Najjar S and Pearlman DM. Neuroinflammation and white matter pathology in schizophrenia: Systematic review. Schizophr Res 2015;161:10212.

17. Calcia MA, Bonsall DR, Bloomfield PS, Selvaraj S, Barichello T, Howes OD. Stress and neuroinflammation: a systematic review of the effects of stress on microglia and the implications for mental illness. Psychopharmacology 2016;233:1637-50.

18. Malan-Muller S, Kilian S, van den Heuvel LL, Soraya B, Laila A, Louise W,etal. A systematic review of genetic variants associated with metabolic syndrome in patients with schizophrenia. Schizophr Res 2016;170:1-17.

19. Brugger SP and Howes OD. Heterogeneity and homogeneity of regional brain structure in schizophrenia: a meta-analysis. JAMA Psychiatry 2017;74:1104-11.

20. Morrison KM, Ramsingh L, Gunn E, Streiner D, Van Lieshout R, Boyle M et al. Cardiometabolic Health in Adults Born Premature With Extremely
Low Birth Weight. Pediatrics 2016; 138.

21. Metcalf SA, Jones PB, Nordstrom T, Markku T, Pirjo M, Jouko M. Serum C-reactive protein in adolescence and risk of schizophrenia in adulthood: a prospective birth cohort study. Brain Behav Immun 2017;59:253-9.

22. Lenz KM, Nugent BM, Haliyur R, McCarthy MM. Microglia are essential to masculinization of brain and behaviour. J Neurosci 2013;33:2761-72.

23. Jauw YW, Zijlstra JM, de Jong D, Vugts DJ, Zweegman S, Hoekstra OS et al. Performance of Zrlabeled-rituximab-PET as an imaging biomarker to assess CD20 targeting: a pilot study in patients with relapsed/refractory diffuse large B cell lymphoma. PLoS ONE 2017;12:e0169828.

24. Lee EE, Hong S, Martin AS, Eyler LT, Jeste DV. Inflammation in schizophrenia: cytokine levels and their relationships to demographic and clinical variables. Am J Geriatr Psychiatry 2017;25:50-61. 